changes sign at each zero then for small $\epsilon(1.5)$ has at least three periodic solutions as stated. [4]

Let

$$
f(x)=A_{3} x^{6}-A_{2} x^{4}+A_{1} x^{2}-A_{0}-C x
$$

where $A_{i}$ and $C$ are constants. Let

$$
I_{k}=\int_{0}^{2 \pi} \sin ^{2} \sigma \cos ^{2 k} \sigma d \sigma, \quad k=0,1,2,3 .
$$

Then $I_{k}>0$ and from (1.7) it follows that

$$
F(\rho)=A_{3} I_{3} \rho^{7}-A_{2} I_{2} \rho^{5}+A_{1} I_{1} \rho^{3}-A_{0} I_{0} \rho .
$$

Let $A_{3} I_{3}=1, A_{2} I_{2}=14, A_{1} I_{1}=49$ and $A_{0} I_{0}=36$. Then all the $A_{i}$ are positive and

$$
F(\rho)=\rho\left(\rho^{2}-1\right)\left(\rho^{2}-4\right)\left(\rho^{2}-9\right) .
$$

Thus $F(\rho)$ vanishes at $\rho=1,2$, and 3 and changes sign at each of these zeros which proves that (1.5) has at least three periodic solutions for small $\epsilon$.

Since (1.2) is assured by $A_{3}>0$ in (1.9) it remains only to show that $f(x)$ satisfies (1.0) and (1.1). Since the $A_{i}$ are already fixed and since $A_{3}>0$ and $A_{0}>0$ it follows easily that if $C$ is large enough then $C x=A_{3} x^{6}-A_{2} x^{4}+A_{1} x^{2}-A_{0}$ has only one negative root, which is near $x=0$ and will be designated by $-x_{1}^{\prime}$, and only one positive root, which is large and will be designated by $x_{1}$. Clearly $f(x)$ now satisfies (1.0) and (1.1) and the example is complete.

With polynomials of higher degree the existence of more periodic solutions can be established. (The argument of Serbin fails in that he does not recognize the restricted range of the monotonicity of $\phi$, in Lefschetz's notation, as stated by Lefschetz [1, p. 193] and to which Serbin refers.)

\title{
REFERENCES
}

1. S. Lefschetz, Lectures on differential equations, Princeton, 1946.

2. N. Levinson and O. K. Smith, A general equation for relaxation oscillations, Duke Math. Journal 9, 382-403 (1942).

3. A. Liénard, Étude des oscillations entretenues, Rev. Gen. d'Élect. 23, 901-946 (1928).

4. H. Poincaré. Les méthodes nouvelles de la mécanique céleste, Vol. 1, Gauthier-Villars, Paris, 1892, Chap. III.

5. H. Serbin, Periodic motions of a nonlinear dynamic system, Q. Appl. Math. 8, 296-303 (1950).

\section{THE COMPRESSIBLE FLOW CORRESPONDING TO A LINE DOUBLET*}

\section{By J. W. CRAGGS (St. John's College, Cambridge)}

Introduction. The use of the hodograph equations for irrotational compressible flow in two dimensions leads, as noticed by Chaplygin ${ }^{1}$ and others, to a method of constructing compressible flow patterns by reference to similar problems in incompressible flow. For certain elementary flows, as for example the flow due to a line source, a line

${ }^{*}$ Received December 11, 1950.

${ }^{1}$ Chaplygin, On gaseous jets, Ann. Sci. Moscow Imperial University, Math.-Phys. section 21, (1904). 
vortex or a line doublet, the method is particularly simple. Ringleb ${ }^{2}$ has investigated these cases, but his diagrams for the doublet do not illustrate what is perhaps one of the most interesting things about the corresponding compressible flow, the type of limit lines and singularities which occur. In the present paper special attention is paid to these points.

The solution in the hodograph plane. For incompressible flow, the complex potential for a line doublet is given by

$$
w=-1 / z, \quad q e^{-i \theta}=d w / d z=1 / z^{2},
$$

where $q$ is the magnitude and $\theta$ the inclination to the real axis of the velocity.

Eliminating $z$ we obtain

whence

$$
w=-q^{1 / 2} e^{-i \theta / 2}
$$

$$
\psi=q^{1 / 2} \sin \theta / 2,
$$

where $\psi$ is the stream function. The two branches of (1) correspond to the two halfplanes, on each side of the doublet axis. If only the upper half-plane is considered the hodograph plane is once covered, but is cut from the origin along $\theta=0$ to $q=1$.

That solution of the hodograph equations of compressible flow which corresponds to $(1)$ is

$$
\psi=q^{1 / 2} f(\tau) \sin \theta / 2,
$$

where

$$
\tau=q^{2} / Q^{2}
$$

$Q$ being the maximum velocity, corresponding to zero density, and

$$
f(\tau)=F(a, b ; 3 / 2 ; \tau)
$$

where $F$ is a hypergeometric function with

$$
a b=-3 \beta / 8, \quad a+b=(1-2 \beta) / 2,
$$

being the reciprocal of $\gamma-1$, where $\gamma$ is the adiabatic index.

The hypergeometric series (3) converges rapidly and is easily calculated for values of $\tau<1$. Near $\tau=1$ the expansion in terms of $1-\tau$ may be used.* Thus the flow pattern in the hodograph plane is found.

Reversion to the physical plane. The coordinates in the physical plane, $x, y$, are given by

and

$$
d x=(d \phi / q) \cos \theta-\left(\rho_{0} / \rho\right)(d \psi / q) \sin \theta
$$

where

$$
d y=(d \phi / q) \sin \theta+\left(\rho_{0} / \rho\right)(d \psi / q) \cos \theta,
$$

$$
\begin{aligned}
q(\partial \phi / \partial q) & =-\left(\rho_{0} / \rho\right)\left(1-M^{2}\right)(\partial \psi / \partial \theta), \\
(\partial \phi / \partial \theta) & =\left(\rho_{0} / \rho\right) q(\partial \psi / \partial q),
\end{aligned}
$$

${ }^{2}$ Ringleb, Exact solutions of the differential equations of an adiabatic gas flow. Z.A.M.M. (1940).

*The form of the alternative expansion may be found in any standard text on hypergeometric functions. 
$M$ being the local Mach number, given by

$$
M^{2}=2 \beta \tau /(1-\tau)
$$

and $\left(\rho_{0} / \rho\right)$ the density ratio, given by

$$
\left(\rho_{0} / \rho\right)^{2}=(1-\tau)^{-2 \beta} .
$$

Equations (2), (4) and (5) lead to expressions for the derivatives of $x$ and $y$ with respect to $q$ and $\theta$. Integration of those expressions then gives

and

$$
x\left(\rho q^{1 / 2} / \rho_{0}\right)=2 \tau(d f / d \tau)\left(\cos \frac{1}{2} \theta-\frac{1}{3} \cos \frac{3}{2} \theta\right)+f \cos \frac{1}{2} \theta
$$

$$
y\left(\rho q^{1 / 2} / \rho_{0}\right)=2 \tau(d f / d \tau)\left(\sin \frac{1}{2} \theta-\frac{1}{3} \sin \frac{3}{2} \theta\right)+f \sin \frac{1}{2} \theta .
$$

The flow in the hodograph plane may be found by plotting streamlines $\psi=$ const. The streamlines in the physical plane are then obtained by substituting corresponding values of $q$ and $\theta$ in (6).

Singularities of the transformation. Now we investigate the singularities of the transformation. The transformation from the hodograph to the physical plane ceases to be regular when

$$
J=\partial(q, \theta) / \partial(x, y)
$$

vanishes or becomes infinite. ${ }^{3}$ The Jacobian reduces to

$$
J=-\left(\rho / \rho_{0}\right)^{2} q^{3} /\left\{q^{2} \psi_{a}^{2}+(1-M)^{2} \psi_{\theta}^{2}\right\}
$$

Zeros of $J$ can occur only when $q=0$ or $\psi_{a}$ or $\psi_{\theta} \rightarrow \infty$. Now $f$ and $d f / d \tau$ are always finite so the last two possibilities are eliminated by (2). As $q \rightarrow 0, f \rightarrow 1$ and $J \rightarrow 0$; this corresponds to a singular point (the point at infinity) in the flow plane, and a branch point in the hodograph plane. The singularity is due to the symmetry of the flow about the $x$ and $y$ axes, which implies the same velocity vector at $(x, y)$ and $(-x,-y)$. This has already been dealt with by the cut in the hodograph plane.

The singularity corresponding to $J \rightarrow \infty$ is of more interest. $J \rightarrow \infty$ implies $q^{2} \psi_{a}^{2}+$ $\left(1-M^{2}\right) \psi_{\theta}^{2}=0$. This can only be satisfied for supersonic velocity, $M>1$. Put $\alpha^{2}=$ $M^{2}=1$, then the condition is

$$
q \psi_{a} \pm \alpha \psi_{\theta}=0
$$

that is

$$
\left(2 \tau f^{\prime}+\frac{1}{2} f\right) q^{1 / 2} \sin \frac{1}{2} \theta \pm \frac{1}{2} \alpha q^{1 / 2} f \cos \frac{1}{2} \theta=0,
$$

whence

$$
\alpha \cot \frac{1}{2} \theta\left(4 \tau f^{\prime} / f\right)+1
$$

The singular line in the hodograph plane, given by (7), is a double loop curve touching the sonic circle, $M=1$, at $\theta=0$, crossing itself at $\theta=180^{\circ}, 4 \tau\left(f^{\prime} / f\right)+1=0,(\tau=$ .451 , approximately) and touching the limit circle, $q=Q$ at $\theta=180^{\circ}$. It is shown in Fig. 1 together with plots of typical streamlines in the hodograph plane.

'Cwass, The breakdown of the hodograph transformation for compressible flow in two dimensions. Camb. Phil. Trans. 44, 1948. 
The limit line in the flow plane may now be found by substitution in (6) of (8). Corresponding to the double point there are zeros of $d x / d \tau$ and $d y / d \tau$ taken along the

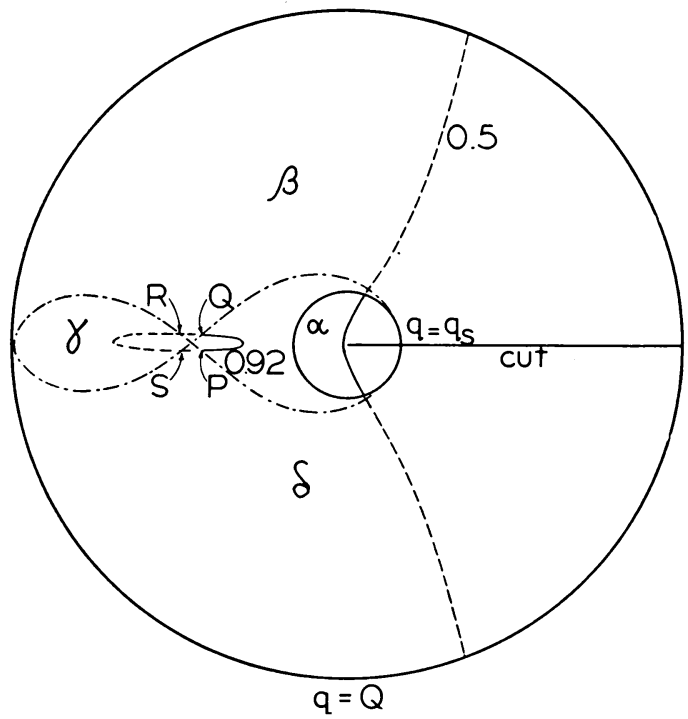

Fig. 1. Hodograph plane.

curve, and each limit line is cusped. It is easily deduced from (2) and (5) that at this point

$$
\psi_{q}=\psi_{\theta}=\phi_{a}=\phi_{\theta}=\psi_{a \theta}=\phi_{a q}=\phi_{\theta \theta}=0
$$

whilst

$$
q \phi_{a \theta}=\left(\rho_{0} / \rho\left(\alpha^{2} \psi_{\theta \theta}\right.\right.
$$

and

$$
q^{2} \psi_{a q}=\alpha^{2} \psi_{\theta \theta},
$$

thus the ratio $d \phi / d \psi$ corresponding to given $d q / d \theta$ at this point is

$$
\frac{d \phi}{d \psi}=\frac{2 \alpha^{2}\left(\rho_{0} / \rho\right) q(d q / d \theta)}{\alpha^{2}(d q / d \theta)^{2}+q^{2}} .
$$

But on a limit line $q d \theta / d q= \pm \alpha$, whence $d \phi / d \psi= \pm \alpha\left(\rho_{0} / \rho\right)$. It follows from (4) that

$$
\frac{d y}{d x}=\frac{\alpha \cos \theta \mp \sin \theta}{\alpha \sin \pm \cos \theta}=\mp \alpha \quad \text { at } \quad \theta=180^{\circ} .
$$

Next, since $\psi q_{\theta}=0$ and $\psi_{\theta a}, \psi_{\theta \theta}$ are of the same sign, we see that the point is an extremum of $\psi$ and hence that lines $\psi=$ const. encircle the point and no streamline passes through it. The singularity is of order two (see ref. 3).

The flow in the physical plane. We may now see the main characteristics of the flow in the physical plane. First we draw in the limit lines, shown chain dotted in Fig. 2. Each of the four branches leaving the point $B$ is the join of two sheets of a Riemann surface, the four sheets of which correspond to the regions $\alpha, \beta, \gamma, \delta$ of Fig. 1. The regions $\beta$ and $\delta$ are separated by the cut $\theta=0$.

The region $\alpha$ corresponds to a sheet covering the whole of the diagram above the lower pair of limit lines, $B P, B Q$. Along $B Q$ it joins a sheet $(\beta)$ bounded by $B Q$ and $B R$. 
The sheet $\gamma$ extends from $B R$ to $B S$ and the fourth, $(\delta)$ bounded by $B S$ and $B P$, is symmetrical to the second, and joins the first $(\alpha)$ along $B P$. A streamline, like that for $\psi=0.92$, which is closed in the hodograph plane, passes over the four sheets in order.

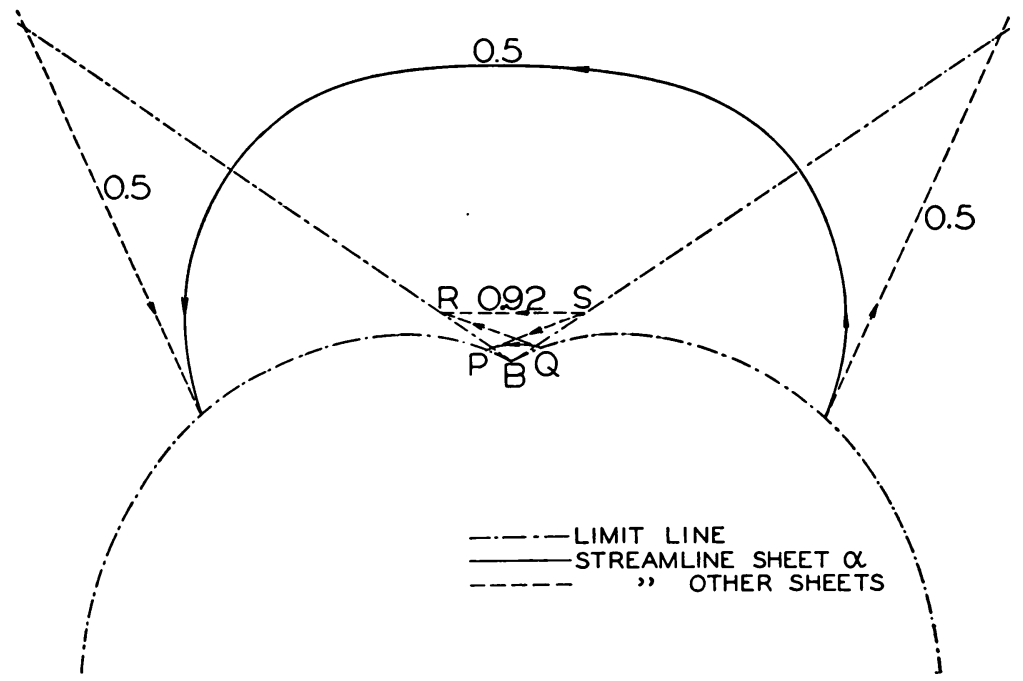

Fig. 2. Physical plane.

The stream line for $\psi=0.5$, which is also shown, never reaches the sheet $\gamma$ but goes off, after reflexion at each point where it meets the limit lines, to infinity with speed at infinity of $Q$.

TABLE I. The Limit Line.

\begin{tabular}{crrr}
$\tau$ & \multicolumn{1}{c}{$\theta$} & \multicolumn{1}{l}{$x$} & \multicolumn{1}{l}{$y$} \\
.16 & 0.00 & 1.278 & .000 \\
.18 & 58.36 & 1.032 & .684 \\
.20 & 87.48 & .715 & .906 \\
.22 & 107.00 & .555 & .986 \\
.24 & 121.00 & .422 & 1.015 \\
.26 & 132.12 & .312 & 1.020 \\
.28 & 141.24 & .230 & 1.014 \\
.30 & 148.48 & .167 & 1.007 \\
.35 & 162.42 & .062 & .997 \\
.40 & 172.36 & .014 & .959 \\
.45 & 179.20 & .001 & .948 \\
.50 & 184.14 & .010 & .955 \\
.55 & 187.42 & .041 & .975 \\
.60 & 190.02 & .095 & 1.003 \\
.65 & 190.42 & .184 & 1.063 \\
.70 & 191.10 & .320 & 1.170 \\
.75 & 192.00 & .589 & 1.314 \\
.80 & 190.44 & .965 & 1.600 \\
.85 & 189.56 & 1.859 & 2.060 \\
.90 & 188.02 & 4.280 & 3.760 \\
.95 & 183.44 & 18.3 & 15.4 \\
1.00 & 180.00 & $+\infty$ & $+\infty$
\end{tabular}


TABLE II. Streamlines

\begin{tabular}{|c|c|c|c|c|c|c|}
\hline \multirow[b]{2}{*}{$\tau$} & \multicolumn{2}{|c|}{$\psi=0.5$} & \multicolumn{2}{|c|}{$\psi=0.9$} & \multicolumn{2}{|c|}{$\psi=0.92$} \\
\hline & $x$ & $y$ & $x$ & $y$ & $x$ & $y$ \\
\hline .00 & & & & & & \\
\hline .02 & .769 & 1.618 & & & & \\
\hline .03 & .92 & 1.347 & & & & \\
\hline .04 & .962 & 1.190 & & & & \\
\hline .05 & .975 & 1.087 & & & & \\
\hline .10 & .961 & .860 & & & & \\
\hline .12 & .952 & .824 & & & & \\
\hline .14 & .945 & .801 & & - & & \\
\hline .16 & .942 & .788 & & & & \\
\hline .18 & .940 & .783 & & & & \\
\hline .20 & .940 & .784 & & & & \\
\hline .22 & .943 & .790 & & & & \\
\hline .24 & .947 & .801 & .065 & 1.017 & & \\
\hline .26 & .954 & .816 & .105 & 1.007 & & \\
\hline .28 & .962 & .836 & .123 & 1.001 & & \\
\hline .30 & .973 & .859 & .131 & .997 & .042 & .983 \\
\hline .35 & 1.009 & .938 & .124 & 1.000 & .068 & .976 \\
\hline .40 & 1.063 & 1.051 & .096 & 1.019 & .054 & .983 \\
\hline .45 & 1.138 & 1.207 & .049 & 1.049 & .019 & .998 \\
\hline .50 & 1.241 & 1.424 & -.018 & 1.091 & -.031 & 1.018 \\
\hline .55 & 1.387 & 1.731 & -.108 & 1.148 & -.904 & 1.042 \\
\hline .60 & 1.599 & 2.181 & -.230 & 1.219 & -.163 & 1.067 \\
\hline .65 & 1.922 & 2.822 & -.397 & 1.318 & -.234 & 1.092 \\
\hline .70 & 2.439 & 3.956 & -.627 & 1.441 & -.126 & 1.091 \\
\hline .75 & 3.333 & 5.986 & -.959 & 1.595 & & \\
\hline .80 & 5.09 & 9.97 & -1.426 & 1.798 & & \\
\hline .85 & 9.25 & 19.59 & -1.766 & 2.022 & & \\
\hline .90 & 22.8 & 51.85 & & & & \\
\hline .95 & 117.4 & 282.6 & & & & \\
\hline .00 & & & & & & \\
\hline
\end{tabular}

For clarity only $\psi=0.5$ and $\psi=0.92$ are shown in the diagrams,

\section{BOOK REVIEWS}

Mécanique des milieux continus et déformables. By Maurice Roy. Gauthier-Villars, Paris, 1950. Volume I, xxii + 363 pp., 2800 fr. Volume II, xii +350 pp., 2300 fr.

M. Roy presents an account of the mechanics of fluids and elastic solids that is based upon the application of thermodynamic principles to continua. The work covers substantially the content of a second year course of lectures at l'École Polytechnique, and the object is to give a synthetic account of the fundamental principles and methods of this mechanics bearing in mind applications to engineering.

Each volume comprises two parts together with extensive appendices; the four parts are entitled "thermodynamics and mechanics of continuous and deformable media", "theory of elasticity", "equilibrium and flow of fluids", and "theory of machines". The principal contents of these parts are described briefly as follows. Part I. "Definitions and fundamentals of deformations"; finite and infinitesimal strains, Eulerian and Lagrangian co-ordinates, acceleration, equation of continuity. "Fundamental thermodynamic principles and equations"; thermodynamic variables, first and second laws of thermodynamics, internal potential, general thermodynamic equation. "General theorems of mechanics"; stability of equilibrium, reciprocal theorems. "Applications to deformable media and elastic solids"; 